\title{
Electromagnetic radiation of oscillating thin films
}

\author{
Alexander $V$. Kharlanov ${ }^{1, *}$ \\ ${ }^{1}$ Volgograd State Technical University, V. I. Lenin Avenue, 28, Volgograd, 400005, Russia
}

\begin{abstract}
This paper deals with the electromagnetic fields generated by charges moving with the membrane. The numerical simulation of electromagnetic radiation of oscillating three-dimensional cell it is carried out. The issues of cell group radiation are also considered. Dependences of the field on distance and time are presented. A hypothesis about possible exchange of information between cells by means of electromagnetic waves is made.
\end{abstract}

\section{Introduction}

Modern electronics development is connected with the issues of miniaturization of electronic devices components and their interintegration. One of the ways to solve this problem is to use thin films and the physical processes occurring in it as separate elements for generation and transformation of electromagnetic signals. A layer of phospholipids can be considered as such film. When placing in water, they form a spherical bilayer. The use of such film as an object of study is very relevant, since the phospholipid bilayer, despite the fact that it can be created artificially, is also the basis for the membranes of most biological cells. This makes it possible to extend the scope of application towards bioelectronics - the field of electronics that considers biologic objects for information processing systems and new devices [1].

A double electrical layer is created at the edge of the film with water. If we consider the film as a membrane, then from the outer and inner side there are known charges of different signs [2]. Mechanical oscillations of the bilayer and, accordingly, the charge can create variable fields in accordance with the electrodynamic codes [3]. Mechanical oscillations of any film can be excited both mechanically (by means of a sound wave [4]) and electromagnetically (by means of a pulsed electromagnetic wave [5]). Also in [6] it is empirically shown that a living cell performs acoustic oscillations even without external influence. Since acoustic oscillations depend on the state of the cell, knowing the parameters of this wave, it is possible to determine the parameters of cell oscillations. It can also be used for signal transformation.

For the first time the possibility of the existence of acoustic vibrations of the cell was predicted by Frohlich. He substantiated the possibility of coherent excitation of plasma membranes of cells or its individual areas at the range of frequencies of $10^{11}-10^{12} \mathrm{~Hz}$, which corresponds to MM waves [7].

* Corresponding author: harlanov_av@mail.ru 
In [8] it is shown that the existence of acoustic waves is in accordance with the experimental data, indicating the presence of waves which velocity corresponds to the velocity of acoustic waves in the cells exposed to EHF radiation. In [9] the waves excited in cell membranes was established experimentally. In [10], this conclusion was confirmed by theoretical analysis performed using real physical parameters of cell membranes. However, the cell membranes of living cells are polarized because there is a potential difference between the outer and inner surfaces of the membranes, which is determined by the existence of an electric field.

Acoustic wave propagation along the membrane causes periodic changes in membrane thickness. The spatial period of membrane thickness change is equal to the acoustic wave length. In polarized dielectric (lipid) membranes, such changes in thickness are accompanied by changes in the electric field, which have the same periodicity as the acoustic waves.

Therefore, when applied to polarized membranes of living organisms, it is more correct to use the term acoustoelectric waves rather than acoustic and electric waves separately. When the wavelength of these waves is slightly shorter than the wavelength of electromagnetic waves, changes in the electrical component cause, as mentioned above, the transformation of this energy into elastic deformations rather than into magnetic field energy.

The papers [11] also consider the generation of electromagnetic waves by biological objects and intercellular interactions. The spectral range of emitted waves is wide: from $\mathrm{kHz}$ to the visible part of the electromagnetic spectrum. In the range of $10^{-2}-10^{4} \mathrm{~Hz}$ at distances from $1 \mathrm{~mm}$ to $1 \mathrm{~m}$ from the body surface, electric fields are registered, the source of which is the bioelectric activity of the corresponding organs and tissues of human body. At frequencies in the range of about $10^{9}-10^{10} \mathrm{~Hz}$, a natural thermal microwave radioemission of the human body begins [12].

There are numerous measurements of electric fields created by biological cells [13 $19]$. The measured frequencies of these fields occur in different ranges $([13-15]-0,01 \div 1$

$\mathrm{Hz},[16-19]-10^{3} \div 10^{8} \mathrm{~Hz}$ ).

In $[17,18]$, variable fields were detected in different crops - saccharomyces cervisiae, monoraphidium griffithii $\left(10^{6}-10^{8} \mathrm{~Hz}\right)$, schizosac-charomyces pombe $\left(10^{6} \mathrm{~Hz}\right)$.

Thus, biological thin films make acoustic oscillations and can emit electromagnetic waves.

\section{The Model}

The oscillating phospholipid bilayer can be represented as a spherical layer of dipoles with variable dipole moments. If the sphere is divided into elementary surfaces $d S$ the elementary dipole moment in spherical coordinates may be written as follows:

$$
d p^{\prime}=h(t) \sigma d S=h(t) \sigma R^{2} \sin \theta_{1} d \theta_{1} d \varphi_{1},
$$

where $R$ is the radius of a layer, $\sigma=q /\left(4 \pi R^{2}\right)$ is the superficial density of a charge on a membrane, $q$ is the charge, $\left(R, \theta_{1}, \varphi_{1}\right)$ the coordinate of an elementary dipole (see fig.1). The length of dipole changes with time according to the expression:

$$
h(t)=h+\eta P_{n}^{m}\left(\cos \theta_{1}\right) \cos \left(m \varphi_{1}\right) \cos \left(\omega_{0} t\right),
$$

where $h$ is the film thickness, $\eta$ is the amplitude of film oscillations, $P_{n}^{m}\left(\cos \theta_{1}\right) \cos \left(m \varphi_{1}\right)$ are the eigenfunctions of the hollow sphere inner space (the amplitude of film oscillations changes according to this law) [20], $\omega_{0}$ is the frequency of acoustic oscillations of the film. 

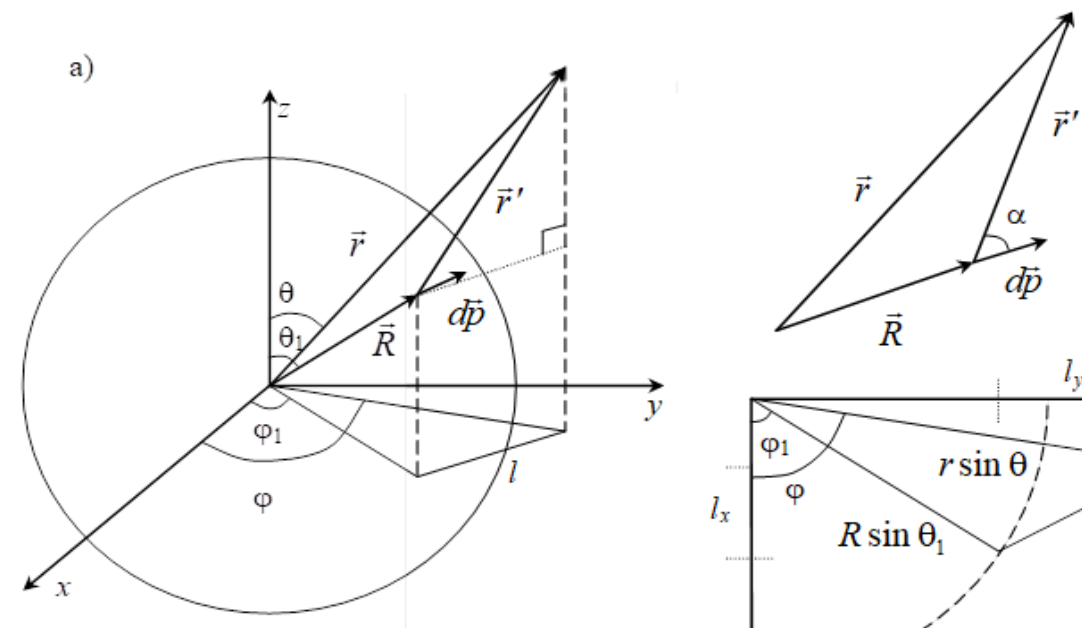

b)

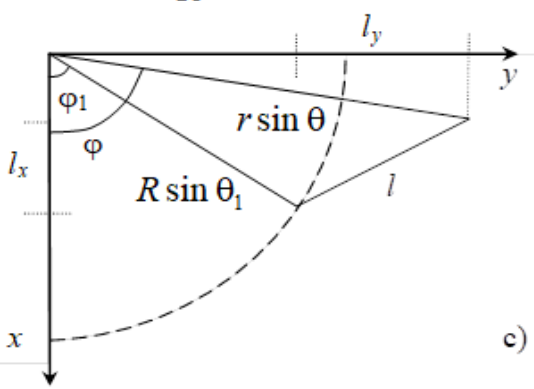

Fig. 1. Spherical thin film.

As the model experiments have shown in the two-dimensional case, in the far-field region the electromagnetic field is very small, and in the near-field zone (region), where $k r^{\prime}=\frac{\omega r^{\prime}}{c}<<1$, and $r^{\prime}>>h$, only the electric field is a significant value. The equation for the intensity of the electric and magnetic fields of dipole radiation in the near-field zone looks as follows [21]:

$$
\mathbf{E}(\mathbf{r})=\frac{1}{4 \pi \varepsilon_{0}}\left[3 \mathbf{n}\left(\mathbf{n} \cdot \mathbf{p}^{\prime}\right)-\mathbf{p}^{\prime}\right] \frac{1}{r^{\prime 3}}, \quad \mathbf{B}=\frac{\mu_{0}}{4 \pi} \frac{[\dot{\mathbf{p}}, \mathbf{n}]}{r^{2}} .
$$

In this equation all values are calculated at the moment of time $\left(t-\frac{r^{\prime}}{c}\right), \mathbf{n}=\frac{\mathbf{r}^{\prime}}{r^{\prime}}$. Then the electric field created by the elementary dipole at the point with coordinates relative to the center of the sphere looks as follows:

$$
d \mathbf{E}(r, \theta, \varphi)=\frac{1}{4 \pi \varepsilon_{0}}\left[3 \mathbf{n} d p^{\prime} \cos \alpha-d \mathbf{p}^{\prime}\right] \frac{1}{r^{\prime 3}}
$$

The projection of the distance from the dipole to the observation point $\left(r^{\prime}\right)$ on the $x 0 y$ plane looks as follows (see fig 1):

$$
l=\sqrt{\left(R \sin \theta_{1}\right)^{2}+(r \sin \theta)^{2}-2 R r \sin \theta_{1} \sin \theta \cos \left(\varphi-\varphi_{1}\right)},
$$

and from a rectangular triangle (fig. 1a)

$$
r^{\prime}=\sqrt{r^{2}+R^{2}-2 R r \cos \theta_{1} \cos \theta\left(1+\operatorname{tg} \theta_{1} \operatorname{tg} \theta \cos \left(\varphi-\varphi_{1}\right)\right)}
$$

On the other hand, figure $1 \mathrm{~b}$ shows:

$$
r^{2}=R^{2}+r^{\prime 2}+2 R r^{\prime} \cos \alpha \Rightarrow \cos \alpha=\frac{r^{2}-R^{2}-r^{\prime 2}}{2 R r^{\prime}} .
$$

Elementary dipole torque projections on the Cartesian coordinate system axis looks as follows: 


$$
\left\{\begin{array}{l}
d p_{x}^{\prime}=d p^{\prime} \sin \theta_{1} \cos \varphi_{1} \\
d p_{y}^{\prime}=d p^{\prime} \sin \theta_{1} \sin \varphi_{1} \\
d p_{z}^{\prime}=d p^{\prime} \cos \theta_{1}
\end{array}\right.
$$

From fig. 1c:

$$
\begin{gathered}
l_{x}=r \sin \theta \cos \varphi-R \sin \theta_{1} \cos \varphi_{1}, \quad l_{y}=r \sin \theta \sin \varphi-R \sin \theta_{1} \sin \varphi_{1}, \\
n_{x}=\frac{l_{x}}{r^{\prime}}, \quad n_{y}=\frac{l_{y}}{r^{\prime}}, \quad n_{z}=\frac{r \cos \theta-R \cos \theta_{1}}{r^{\prime}},
\end{gathered}
$$

By substituting these expressions in formula (1) and integrating it over the entire surface of the sphere, it is possible to find the field created by the oscillating film.

\section{Results and Discussion}

The following parameters have been taken for the calculations: system charge $q=10^{-13} \mathrm{~K} 1$ [22], film thickness $h=10^{-8} \mathrm{~m}$, film oscillation amplitude $\eta=10^{-10} \mathrm{~m}$, film radius $R=10^{-5} \mathrm{~m}$, frequency of oscillations $\omega_{0}=10^{8} \mathrm{~s}^{-1}$ (frequency of acoustic oscillations [20, 23]).

Figure 2 shows diagrams of the dependence of electric field intensity projections in the near-field zone on the coordinate. The obtained values were calculated at the moment of time $t=10^{-7} \mathrm{~s}$ with the distance of $r=1 \mathrm{~mm}$ under the angle of observation $\varphi=\pi / 2 \mathrm{rad}$, for the lowest type of spherical membrane oscillation $(n=1, m=0)$. It can be seen that for this type of oscillation, the resulting field is highly dependent on the direction of the oscillation (it does not depend on the value of $\varphi$, since the membrane thickness does not change).

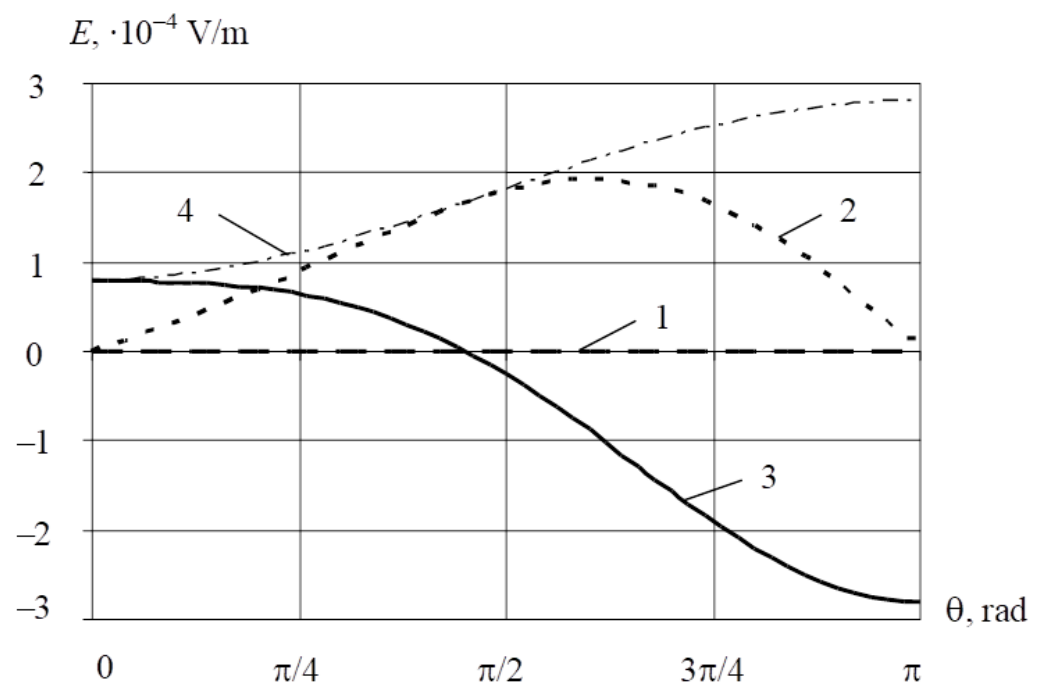

Fig. 2. Field intensity of the film at a distance $r=1 \mathrm{~mm}, \varphi=\pi / 2 \mathrm{rad}\left(1-E_{x}, 2-E_{y}, 3-E_{z}, 4\right.$ $-E, n=1, m=0)$. 


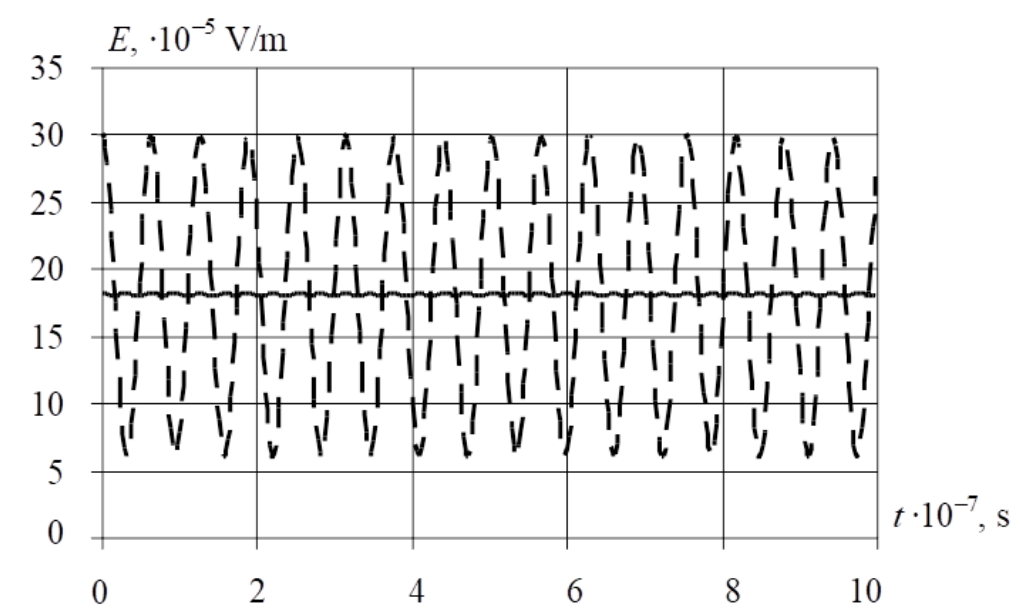

Fig. 3. Dependence of the electrical field intensity of the film on time $\varphi=\pi / 2 \mathrm{rad}, n=1, m=0$ (solid line $-\theta=\pi / 2 \mathrm{rad}$, dotted line $-\theta=0 \mathrm{rad}$ ).

Figure 3 shows the dependence of the electric field created by the lowest type of oscillation on time for two points $-(1 \mathrm{~mm}, 0 \mathrm{rad}, \pi / 2 \mathrm{rad})$ and $(1 \mathrm{~mm}, \pi / 2 \mathrm{rad}, \pi / 2 \mathrm{rad})$. It is clearly visible how much the field changes (several times), despite the fact that the dipole moment of the film changes slightly (only $1 \%$ ).

Fig. 4 shows the field for oscillation for $n=1, m=1$. Observation angle $\theta=\pi / 2$. All other parameters are the same as in fig. 2.

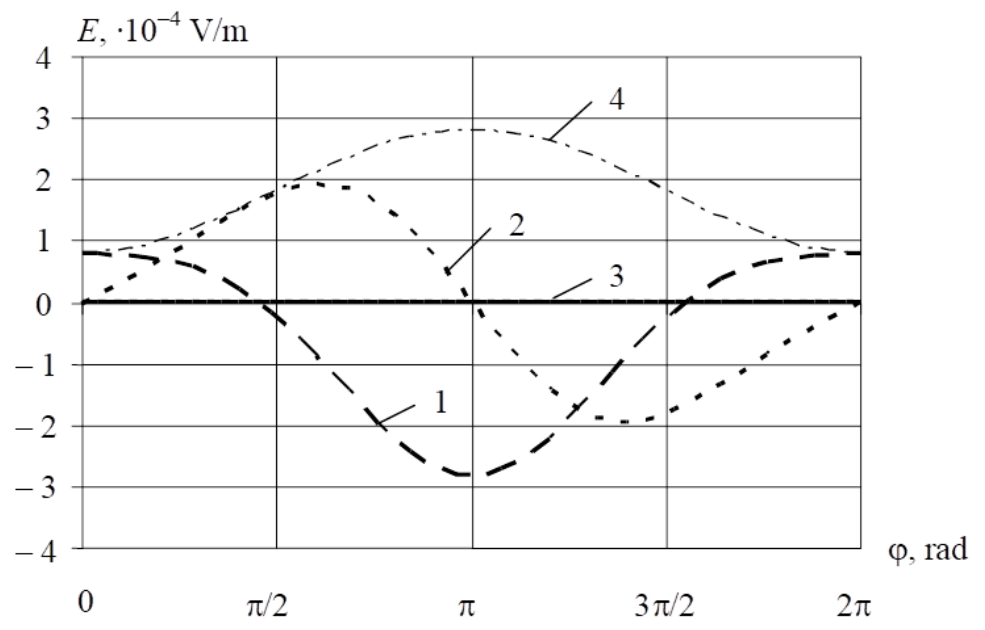

Fig. 4. Field intensity generated by the film at a distance of $r=1 \mathrm{~mm}, \theta=\pi / 2 \operatorname{rad}\left(1-E_{x}, 2-\right.$ $\left.E_{y}, 3-E_{z}, 4-E, n=1, m=1 . t=10^{-7} \mathrm{~s}\right)$.

The magnetic field induction created by the cell has a small value (for example, at a distance of $\left.1 \mathrm{~mm} B \sim 10^{-16} \mathrm{Tl}\right)$. 
These results are obtained for a zero conductivity medium. Since water can generally carry electrical current, it is necessary to summarise the results with regard to the conductive properties of the substance. For this purpose, a complex dielectric permittivity is required:

$$
\widetilde{\varepsilon}=\varepsilon \varepsilon_{0}-j \frac{\sigma}{\omega},
$$

where $\sigma$ is the conductivity of water, $j$ is the imaginary unit. In this case, the penetration depth will be equal to [24]:

$$
d=\sqrt{\frac{2}{\omega^{2} \mu \mu_{0}\left[\sqrt{\left(\varepsilon \varepsilon_{0}\right)^{2}+(\sigma / \omega)^{2}}-\varepsilon \varepsilon_{0}\right]}},
$$

where the relative dielectric permittivity $\varepsilon$ and conductivity of water $\sigma$ depend on the frequency of the electromagnetic field.

As can be seen from Figure 5, with conductivity values corresponding to the frequencies of acoustic oscillations, the depth of penetration has a value of a row of meters, and at a distance of $1 \mathrm{~mm}$ the electric field intensity decreases by only a few percent.

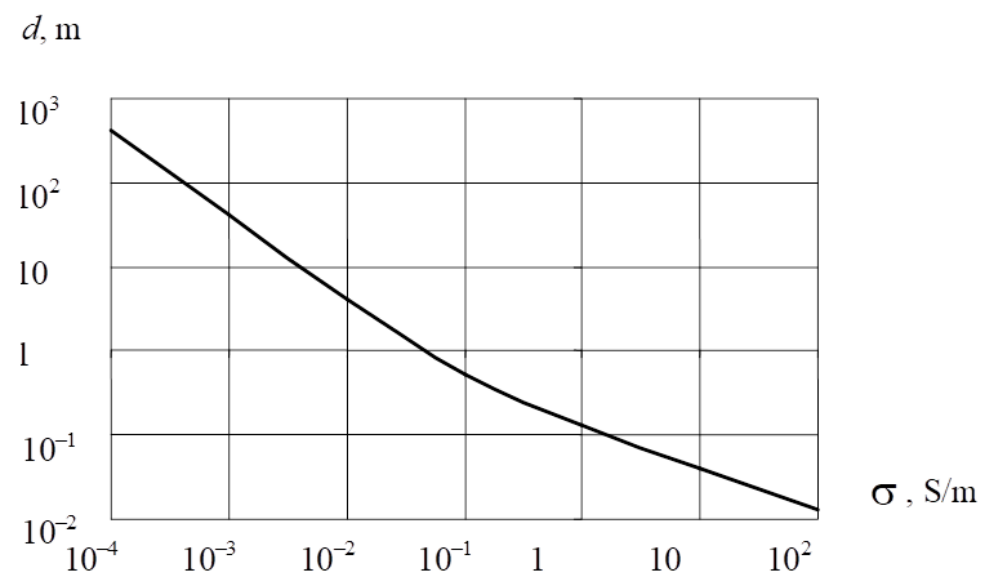

Fig. 5. Dependence of penetration depth on the conductivity of the medium.

Thus, the fields created by oscillating thin films can be used as a source of information about the environment and the film itself, since their parameters influence the type of oscillation. The films can also be used to convert signals by exciting the acoustic oscillations of the films with an external signal.

\section{References}

1. C. Nicolini, From neural chip and engineered biomolecules to bioelectronic devices: an overview, Biosensors and Bioelectronics, 10(1-2), pp. 105 - 127 (1995)

2. A.B. Rubin, Biophysics (Higher school, Moscow, 1987)

3. A. G. Shein, A. V. Kharlanov, Dipole representation of the membrane, Biomedical technologies and radioelectronics, 5, pp. $15-19$ (2007) 
4. P.V. Zinin, J.S. Allen, Deformation of biological cells in the acoustic field of an oscillating bubble, Physical Review E, 79(2 Pt 1):021910 (2009)

5. A. V. Kharlanov, Forced Acoustic Oscillations of Biological Cell, Bioelectromagnetics, 38(8), pp. 613 - 617 (2017)

6. A. E. Pelling, S. Sehati, E. B. Gralla, J. S. Valentine, J. K. Gimzewski, Local Nanomechanical Motion of the Cell Wall of Saccharomyces cerevisiae, Science, 305, 5687, pp. 1147-1150 (2004)

7. H. Frohlich, Bose condensation of strongly excited longitudinal electric modes, Phys. Lett. 26 A (1968)

8. M. B. Golant, Acousto-electric waves in cell membranes of living organisms - a key problem for understanding of mm-waves interaction with living organisms, Biological aspects of low intensity millimeter waves, pp. 229 - 249 (1994)

9. K. J. Blinovska, W. Lech, A. Wittlin, Cell membranes as a possible site of Frohlich Coherent Oscillation, Phys. Letters, v. 109 A 3, pp. 124 - 126 (1985)

10. M. Cifra, J. Z. Fields, A. Farhadi, Electromagnetic cellular interactions, Progress in Biophysics and Molecular Biology, 105, pp. 223 - 246 (2011)

11. V.S. Bystrov, Dynamics of systems with hydrogen bonds, Biomedical radioelectronics, v. 3 , pp. $34-40$ (2000)

12. H. Frohlich, Coherent electric vibrations in biological systems and the cancer problem, IEEE Transactions on Microwave Theory and Techniques, 26(8), pp. 613 - 618 (1978)

13. J. Z. Gao, H. X. Dai, H. Chen, J. Ren, W. Yang, Study on the oscillating phenomena of electrical potential across a liquid membrane, Chinese Chemical Letters.; 18(3), pp. $309-312(2007)$

14. J. H. Miller, D. Nawarathna, D. Warmflash, F. A. Pereira, W. E.Brownell, Dielectric properties of yeast cells expressed with the motor protein prestin, Journal of Biological Physics. 31 (3-4), pp. 465 - 475 (2005)

15. R. P. Rastogi, R. C. Srivastava, Interface-mediated oscillatory phenomena, Advances in Colloid and Interface Science, 93 (1-3), pp. 1 - 75 (2001)

16. H. A. Pohl, Interactions between Electromagnetic Fields and Cells, AC Field effects of and by Living Cells. Plenum press, New York and London, pp. 437 - 458 (1985)

17. R. Haolzel, I. Lamprecht, Electromagnetic fields around biological cells, Neural Network World. 3, pp. 327 - 337 (1994)

18. R. Haolzel, Electric activity of non-excitable biological cells at radiofrequencies, Electro- and magnetobiology, 20 (1), pp. 1 - 13 (2001)

19. A. H. Jafary-Asl, C. W. Smith, Biological dielectrics in electric and magnetic fields, in Ann. Rep. Conf. Electrical Insulation and Dielectric Phenomena. IEEE Publ.; pp. 350 355 (1983)

20. A. G. Shein, A. V. Harlanov, Cross acoustic oscillations of the cell and the influence of electromagnetic waves of low intensity on it, Biomedical technologies and radioelectronics, v. 4, pp. $10-15$ (2006)

21. A. M. Fedorchenko, Theoretical physics. Classical electrodynamics (Vyshcha shk., K, 1988)

22. O.V. Betsky, N.N. Lebedeva, Modern ideas about the mechanisms of low-intensity millimeter waves influence on biological objects, Millimeter waves in biology and medicine, v. 3, pp. 5-19 (2001)

23. A. V. Kharlanov, Electromagnetic waves and vibrations of biological objects (VSTU, Volgograd, 2012)

24. S. I. Baskakov, Electrodynamics and propagation of radio waves (Higher Shk, Moscow, 1992) 